\title{
Atrial Fibrillation: Diagnosis, Treatment and Outcomes- A Case Study
}

Md. Sohel Khan ${ }^{1 *}$, Md. Shahabuddin Khan ${ }^{2}$, Md. Hanif Hossain ${ }^{3}$, A. K. Al Miraj ${ }^{4}$, H. N. Ashikur Rahaman ${ }^{5}$ Md. Magfur Rahman ${ }^{6}$, Mahbubul Islam Khandoker ${ }^{7}$, Md. Enamul Hoque ${ }^{7}$, Muhammed Zafar Iqbal ${ }^{7}$

\footnotetext{
${ }^{1}$ Registrar, Department of Cardiology, Al-Helal Specialized Hospital Ltd. 150 Rokeya Sarani, Senpara Parbata, Mirpur, Dhaka-1216, Bangladesh

${ }^{2}$ Professor, Department of Cardiology (Specialist in Medicine \& Cardiology), MBBS (DMC), MCPS (Med), MD (Card), Ph.D, MRCP (Ireland), FRCP (UK), D.Sc (Cardiology), Fellow Interventional Cardiology (India), Al-Helal Specialized Hospital Ltd. 150 Rokeya Sarani, Senpara Parbata, Mirpur, Dhaka-1216, Bangladesh

${ }^{3}$ Medical officer, Department of Cardiac Surgery, Bangabandhu Sheikh Muzib Medical University, Dhaka, Bangladesh

${ }^{4}$ Research Assistant, Department of Vascular Surgery, Bangabandu Sheikh Mujib Medical University, Dhaka, Bangladesh

${ }^{5}$ Registrar, Dept. of Clinical Oncology, Enam Medical College \& Hospital, Savar, Dhaka, Bangladesh

${ }^{6}$ Cardiac Surgeon \& Consultant (Cardiologist \& Diabetologist), Department of Cardiac Surgery, Bangabandu Sheikh Mujib Medical University, Dhaka, Bangladesh

${ }^{7}$ Medical officer, Department of Urology, Bangabandhu Sheikh Muzib Medical University, Dhaka, Bangladesh
}

DOI: $10.36347 /$ sjmcr.2020.v08i12.008

| Received: 05.12.2020 | Accepted: 16.12.2020 | Published: 19.12.2020

*Corresponding author: Md. Sohel Khan

Abstract

Atrial fibrillation is the most common cardiac arrhythmia. It impairs cardiac function and increases the risk of stroke. The incidence of atrial fibrillation increases with age. Key treatment issues include deciding when to restore normal sinus rhythm, when to control rate only, and how to prevent thromboembolism. Rate control is the preferred management option in most patients. Rhythm control is an option for patients in whom rate control cannot be achieved or who have persistent symptoms despite rate control. The current recommendation for strict rate control is a resting heart rate of less than 80 beats per minute. However, one study has shown that more lenient rate control of less than 110 beats per minute while at rest was not inferior to strict rate control in preventing cardiac death, heart failure, stroke, and life-threatening arrhythmias. Anticoagulation therapy is needed with rate control and rhythm control to prevent stroke. Atrial fibrillation prevalence has been on the rise. The risk of stroke is 5-times higher in a patient with known atrial fibrillation compared to the general public. It is estimated that $19.6 \%$ of patients over the age of 65 will have apparent atrial fibrillation by 2030 . The most feared side effect of atrial fibrillation is an acute stroke, which can lead to severe morbidity and mortality. It has been shown that $60 \%$ of strokes secondary to atrial fibrillation can be avoided with the use of anticoagulants. Using the CHADs-2-VASc score to evaluate patients with atrial fibrillation is a helpful guide for the management of these patients with the ultimate goal of preventing stroke. These AF classifications are not mutually exclusive and it is common for patients with one type of AF to exhibit overlapping features of another type. These classifications are relevant clinically with respect to outcomes and prognosis with rhythm-controlling treatment strategies. Atrial fibrillation is commonly associated with other supraventricular arrhythmias, namely atrial flutter and focal atrial tachycardia. Ongoing and future epidemiological, translational, and clinical studies may provide insight into these unanswered questions and improve clinical outcomes in patients with AF.

Keywords: Atrial fibrillation (AF), ablation; surgery, epidemiology, risk factors, prognosis, pathophysiology.

Copyright $\odot 2020$ The Author(s): This is an open-access article distributed under the terms of the Creative Commons Attribution 4.0 International License (CC BY-NC 4.0) which permits unrestricted use, distribution, and reproduction in any medium for non-commercial use provided the original author and source are credited.

\section{INTRODUCTION}

Atrial fibrillation (AF) is a common heart rhythm disorder caused by degeneration of the electrical impulses in the upper cardiac chambers (atria) resulting in a change from an organized heart rhythm to a rapid, chaotic rhythm. The resulting arrhythmia is often rapid and irregular with no discernible pattern (known as irregularly irregular). The disrupted rhythm occurs because of the unpredictable conduction of disordered impulses across the electrical bridge, called the atrioventricular (AV) node, to the lower cardiac chambers (ventricles). The arrhythmia also results in ineffectual atrial contractions affecting cardiac output and vulnerability to blood clot (thrombus) formation that can result in stroke events. The incidence and prevalence of atrial fibrillation are increasing because of both population ageing and an age-adjusted increase in incidence of atrial fibrillation. Deciding between a rate control or rhythm control approach depends on patient age and comorbidities, symptoms and haemodynamic consequences of the arrhythmia, but 
either approach is acceptable. Digoxin is no longer a first-line drug for rate control: beta-blockers and verapamil and diltiazem control heart rate better during exercise. Anti-arrhythmic drugs have only a 40\%-60\% success rate of maintaining sinus rhythm at 1 year, and have significant side effects. Atrial fibrillation is the most common type of cardiac arrhythmia. It is due to abnormal electrical activity within the atria of the heart, causing them to fibrillate. It is characterized as a tachyarrhythmia, which means that the heart rate is often fast. This arrhythmia may be paroxysmal (less than seven days) or persistent (more than seven days). Due to its rhythm irregularity, blood flow through the heart becomes turbulent and has a high chance of forming a thrombus (blood clot), which can ultimately dislodge and cause a stroke. Atrial fibrillation is the leading cardiac cause of stroke. Risk factors for atrial fibrillation include advanced age, high blood pressure, underlying heart and lung disease, congenital heart disease, and increased alcohol consumption. Symptoms vary from asymptomatic to symptoms such as chest pain, palpitations, fast heart rate, shortness of breath, nausea, dizziness, diaphoresis (severe sweating), and generalized fatigue. Although atrial fibrillation may be a permanent disease, various treatments and risk modifying strategies have been developed to help reduce the risk of stroke in patients that remain in atrial fibrillation. Treatments include anticoagulation, rate control medication, rhythm control medication, cardioversion, ablation, and other interventional cardiac procedures [1-3].

\section{Etiology}

There are many causes of atrial fibrillation (AF), but it shares a strong association with other cardiovascular diseases. The commonly encountered causes include:

1. Advanced age

2. Congenital heart disease

3. Underlying heart disease - valvular disease, coronary artery disease, structural heart disease, atrial ischemia

4. Increased alcohol consumption

5. Hypertension - systemic or pulmonary

6. Endocrine disorders - diabetes, pheochromocytoma, and hyperthyroidism

7. Genetic factors

8. Neurologic disorders - subarachnoid hemorrhage or stroke

9. Hemodynamic stress - mitral or tricuspid valve disease, left ventricular dysfunction, pulmonary embolism

10. Obstructive sleep apnea

11. Inflammation - myocarditis, and pericarditis

Any condition that leads to inflammation, stress, damage, or ischemia affecting the anatomy of the heart can result in the development of atrial fibrillation. In some cases, the cause is iatrogenic.[4] Atrial fibrillation is referred to as recurrent when a patient has two or more episodes. The three patterns of atrial fibrillation include:

Paroxysmal AF: If recurrent AF reverts spontaneously, it is called paroxysmal AF. Here, the episodes terminate spontaneously within seven days. In younger patients, paroxysmal AF has been commonly found to be secondary to electrically active foci within the pulmonary veins. Elimination of these foci is found to be effective in treating this type of $\mathrm{AF}$ since it eliminates the trigger for such episodes.

Persistent AF: If recurrent AF persists, needing either pharmacological or electrical cardioversion, it is called persistent AF. In this case, the episodes last more than seven days, and if it is associated with a rapid and uncontrolled ventricular rate, it may lead to electrical remodeling in the cardiac myocytes causing dilated cardiomyopathy. This type of AF may present as the first episode or as a result of recurrent episodes of paroxysmal AF.

Long-standing persistent AF: AF that has been present for more than 12 months, either due to the failure of initiation of pharmacological intervention or failure of cardioversion.

Permanent AF: It is the type where a decision has been made to abort all therapies because the rhythm is unresponsive.

\section{Epidemiology:}

Atrial fibrillation is the most commonly encountered cardiac rhythm disorder. The prevalence of atrial fibrillation has been increasing worldwide. It is known that the prevalence of atrial fibrillation generally increases with age. It has been estimated that the number of individuals with atrial fibrillation will double or triple by the year 2050. Although the worldwide prevalence of atrial fibrillation is approximately $1 \%$, it is found in approximately $9 \%$ in individuals over the age of 75 . At the age of 80 , the lifetime risk of developing atrial fibrillation jumps to $22 \%$. In addition, atrial fibrillation has more commonly been associated with males and seen more often in whites as compared to blacks $[5,6]$.

\section{Pathophysiology}

There are a wide variety of pathophysiological mechanisms that play a role in the development of atrial fibrillation (AF); however, it is the cardiac remodeling that accounts for most of them. Cardiac remodeling, particularly of atria, results in structural and electrical changes that eventually become the cause of deranged rhythm in AF. Structural remodeling is caused by the changes in myocytes and the extracellular matrix, and fibrous tissue deposition also plays a major role in some etiologies. On the other hand, tachycardia and shortening of the refractory period lead to electrical remodeling. Most commonly, hypertension, structural, 
valvular, and ischemic heart disease illicit the paroxysmal and persistent forms of atrial fibrillation, but the underlying pathophysiology is not well understood. Some research has shown evidence of genetic causes of atrial fibrillation involving chromosome 10 (10q22-q24) that consists of a mutation in the gene, alpha-subunit of the cardiac Ik5, which is responsible for pore formation. This is a gain of function mutation, allowing for more pores, increasing the activity within the ion channels of the heart, and thus affecting the stability of the membrane and reducing its refractory time [1]. Most cases of atrial fibrillation are non-genetic and relate to underlying cardiovascular disease. Typically, an initiating trigger excites an ectopic focus in the atria, most commonly around the area of the pulmonary veins, and allows for an unsynchronized firing of electrical impulses leading to fibrillation of the atria. These impulses are irregular, and pulse rates can vary tremendously. Overall, atrial fibrillation leads to a turbulent and abnormal flow of blood through the heart chamber, decreasing the heart effectiveness to pump blood while increasing the likelihood of thrombus formation within the atria, most commonly the left atrial appendage.

\author{
Triggers for AF include \\ 1. Atrial ischemia \\ 2. Inflammation \\ 3. Alcohol and illicit drug use \\ 4. Hemodynamic stress \\ 5. Neurological and endocrine disorders \\ 6. Advanced age \\ 7. Genetic factors
}

\section{History and Physical}

History and physical exam are crucial for diagnosing and risk stratifying patients with atrial fibrillation. The presentation of AF can range from asymptomatic to devastating complications such as cardiogenic shock and ischemic stroke. A complete history should focus on symptoms such as palpitations, chest pain, shortness of breath, increased lower extremity swelling, dyspnea on exertion, and dizziness. In addition, history is imperative in identifying risk factors such as hypertension, history of valvular, structural, or ischemic heart disease, obstructive sleep apnea, obesity hypoventilation syndrome, smoking, alcohol intake, illicit drug use, history of rheumatic fever/heart disease, history of pericarditis, and hyperlipidemia. Initial evaluation of any patient presenting with features of AF should include the assessment for hemodynamic instability. Assessment of patients with existing AF includes questions regarding:

1. Duration and frequency of symptoms

2. History of triggers

3. Previously successful modes of termination

4. The use of anti-arrhythmic drugs

5. Antecedent cardiac diseases
A physical exam should always begin with the assessment of airway breathing and circulation as it is going to affect the decision making regarding management. On general physical examination, patients may be tachycardiac with an irregularly irregular pulse. The heart rate usually ranges from 110/min to 140/min. Extremities should be evaluated for edema, peripheral pulses in both upper and lower extremities, and integumentary signs of peripheral vascular disease (PVD) such as hair loss and skin breakdown. The physical exam should focus on identifying the cause of AF. For instance, examining the neck of the patient may give some clues regarding carotid artery disease or thyroid problems. The pulmonary examination may reveal signs of heart failure in the form of rales, and the presence of wheeze may indicate antecedent pulmonary diseases such as asthma and chronic obstructive pulmonary disease (COPD). A cardiovascular exam should consist of careful auscultation of all four cardiac posts and palpation of apical impulse, as this would be crucial in diagnosing an underlying valvular pathology. An abdominal exam should consist of palpating the aorta and listening for abdominal bruits. Moreover, hepatomegaly and abdominal distension may indicate heart failure. Subsequently, a careful examination of the central and peripheral nervous system may reveal signs of transient ischemic attack or cerebrovascular accident.

\section{Evaluation}

Aside from a detailed history and examination, the ECG is critical in making the diagnosis of atrial fibrillation. On ECG, atrial fibrillation presents with the typical narrow complex "irregularly irregular" pattern with no distinguishable p-waves. Fibrillary waves may be seen or they may be absent. The ventricular rate usually ranges between 80 and 180/min. Laboratory work is required to evaluate for the causes of atrial fibrillation, for example, a complete blood count (CBC) for infection, basic metabolic panel (BMP) for electrolyte abnormalities, thyroid function tests to evaluate for hyperthyroidism, and a chest $\mathrm{x}$-ray to evaluate the thorax for any abnormality. Several cardiac diseases are associated with AF; therefore it is essential to send cardiac biomarkers and B-type natriuretic peptide (BNP) to preclude the underlying cardiac disorder. Interventions such as cardiac catheterization may also be needed in certain cases if the history and physical findings are suggestive. It is imperative to evaluate the patient for pulmonary embolism (with the d-dimers test or spiral CT scan) because the right heart strain can lead to atrial malfunctioning and atrial fibrillation. The patient should be risk-stratified for pulmonary embolism using the PERC and/or Wells criteria. In addition, a transesophageal echocardiogram should be done for these patients to evaluate for atrial thrombus secondary to atrial fibrillation and heart structure. It is important to note that Transesophageal echocardiogram (TEE) should always be done prior to cardio version in these patients to minimize the risk of stroke $[7,8]$. 


\section{Signs and Symptoms}

The clinical manifestations of $\mathrm{AF}$ are variable, although fatigue is the most common symptom. Often, the symptoms are attributable to the rapid ventricular response. However, even when the ventricular response is controlled, symptoms can occur from loss of AV synchrony or atrial systole This is particularly important for patients with left ventricular dysfunction (CHF) and impaired diastolic filling (mitral stenosis, hypertrophic and restrictive cardiomyopathy) .That said, some patients with $\mathrm{AF}$ are genuinely asymptomatic, even at rapid heart rates for unclear reasons. More often, however, patients report nonspecific symptoms such as fatigue, dyspnea, dizziness, and diaphoresis. Palpitations are a common feature. Occasionally, patients present with extreme manifestations of hemodynamic compromise, such as chest pain, pulmonary edema, or syncope. Atrial fibrillation is present in $10 \%$ to $40 \%$ of patients with a new thromboembolic stroke [15].

\section{Case STudy \\ Treatment / Management}

The management of atrial fibrillation in the acute setting depends on the hemodynamic stability and risk stratification. In cases where the patient is hemodynamically unstable, it is recommended to carry out immediate cardioversion with anticoagulant therapy. Although TEE is recommended before any cardioversion; however, if the patient is hemodynamically unstable due to a rapid ventricular response, cardioversion may be indicated without prior TEE. If there is evidence of rapid ventricular response, a beta-blocker or calcium-channel blocker should be commended for rate control. These options can be used in the intravenous (IV) form for rapid response. Usually, a bolus is administered to the patient and then started on a drip if symptoms do not resolve. Digoxin can be considered for rate control but is not advised as a first-line agent pertaining to its adverse effects and tolerance. Amiodarone can also be given as a rhythm control agent but is also not a first-line option in the acute setting. In any case, if the decision to start amiodarone is made, cardiology should be consulted before its administration. In the case of preexisting atrial fibrillation, the patient should be riskstratified using the CHADs-2-Vasc score, which is helpful in estimating the risk of stroke per year. If the patient receives a 0 score, it is considered "low-risk" and anticoagulation is not recommended in such cases. If the patient receives a score of 1 , it falls in the "lowmoderate" risk category; the providers should consider anticoagulant or antiplatelet therapy. If the patient receives a score of greater than 2 , they are in the "moderate-high" risk category, and anticoagulation therapy is indicated [2]. Rate or rhythm control should also be given to the patient; medications such as betablockers, calcium channel blockers, amiodarone, dronedarone, and digoxin are available options. HASBLED is also a scoring system that can be used to assess the risk of bleeding for the patient. This is a good indicator of bleeding risk for a patient that is being considered for anticoagulation. Non-pharmacological therapy includes ablation therapy. Pacemaker placement is considered in severe cases resulting in heart failure in atrial fibrillation [9-11].

\section{Current guidelines}

1. In patients with AF and elevated CHA2DS2-VASc score of 2 or more, oral anticoagulation is recommended.

2. Females with the absence of AF risk factors and males with CHA2DS2-VASc of 1 or 0 have a low stroke risk.

3. Non-vitamin $\mathrm{K}$ oral anticoagulants (apixaban, dabigatran, edoxaban, and rivaroxaban) are recommended over warfarin, except for patients with moderate to severe MS with a mechanical heart valve in place.

4. In all patients with AF, the CHA2DS2-VASc score is recommended to assess stroke risk.

5. Obtain renal and liver function before initiating non-vitamin $\mathrm{K}$ oral anticoagulants.

6. Aspirin is not recommended in patients with low CHA2DS2-VASc scores.

7. Idarucizumab is recommended for dabigatran reversal if there is an urgent procedure or bleeding. Andexanet alfa is recommended for reversal of rivaroxaban and apixaban associated bleeding.

8. Percutaneous left atrial appendage occlusion is recommended in AF patients with a risk of stroke who have contraindications to long term anticoagulation.

9. If the duration of AF is less than 48 hours or if time is unknown, start anticoagulation and maintain INR between 2-3 or commence a factor Xa inhibitor for at least three weeks before and at least four weeks after cardioversion.

10. Catheter ablation is an option in patients with a low ejection fraction.

11. Recommend weight loss in obese patients with AF.

\section{Differential Diagnosis}

History and examination play a crucial role in distinguishing various causes of atrial fibrillation. Different presentations on electrocardiography are a cornerstone in establishing the etiology of AF. Differential diagnoses include:

1. Atrial flutter - However, atrial fibrillation has a distinctive irregularly irregular rhythm with absent P-waves, whereas atrial flutter has a regularly irregular rhythm with absent $\mathrm{P}$-waves.

2. Atrial tachycardia

3. Multifocal atrial tachycardia

4. Wolf-Parkinson-White syndrome

5. Atrioventricular nodal reentry tachycardia.

\section{Staging:}

\section{Classification of atrial fibrillation:}

1. Paroxysmal AF is when the episodes terminate spontaneously or with treatment within 7 days. But they may recur with an unpredictable frequency 
2. Persistent $\mathrm{AF}$ is when the $\mathrm{AF}$ is continuous and lasts for more than 7 days, and fails to terminate spontaneously.

3. Long-standing AF is when the continuous AF lasts more than 12 months

4. Permanent is when AF is accepted and no further treatments are attempted to restore or maintain normal sinus rhythm

5. Non-valvular AF occurs in the absence of rheumatic mitral valve disease, mitral valve repair or a prosthetic heart valve.

\section{CHA2DS2-VASc score}

1. Heart failure - 1

2. Hypertension -1

3. Age More than $75-2$

4. Diabetes - 1

5. Stroke, TIA - 2

6. PVD - 1

7. Age 65-74 - 1

8. Female sex -1

\section{Prognosis}

$\mathrm{AF}$ is associated with a high risk of thromboembolism and death. Evidence shows that rhythm control does not offer a survival advantage over rate control. Patients with $\mathrm{AF}$ have multiple admissions and anticoagulation related complications over their lifetime. The risk of stroke is ever-present, and the overall quality of life of patients is poor. Finally, the management of atrial fibrillation is prohibitively expensive, with most of the financial burden born by the patient.

\section{Complications}

The major side effect of atrial fibrillation is a stroke. Cerebral vascular accident (CVA) can lead to severe morbidity and mortality. CVA risk can be reduced significantly by anticoagulation with adjunct rate/rhythm therapy. Other complications include heart disease and heart failure secondary.

\section{Pearls and Other Issues}

Atrial fibrillation is a commonly encountered disease that affects many individuals. Advancing age is associated with increased prevalence, with the most catastrophic complication being an acute ischemic stroke. Due to the irregularity of the atria, blood flow through this chamber becomes turbulent, leading to thrombus formation. The most common site for this embolus is the atrial appendage. The thrombus can dislodge and embolize to the brain and other parts of the body. It is essential for the patient to seek medical care immediately if they are experiencing chest pain, palpitations, and shortness of breath, severe sweating, or extreme dizziness.

\section{Enhancing Healthcare Team Outcomes:}

Atrial fibrillation is a chronic disorder that can seriously affect the quality of life and costs healthcare billions of dollars each year. Each year, thousands of patients develop stroke and other embolic phenomena, leading to significant disability. The disorder is best managed by an interprofessional team to improve outcomes. A nurse should be dedicated to the monitoring of anticoagulation profile and ensuring the INR is within the therapeutic range. In addition, the nurse should immediately communicate with the team if there are signs of a stroke or other embolic phenomenon. The nurse has to educate the patient on medication compliance for hypertension, coronary disease, and to ensure follow-up at regular intervals. Finally, the patient should be educated about the symptoms of a stroke and when to return to the emergency department [12]. The pharmacist should inform the patient on the different anticoagulants, their benefits, and the adverse risk profile. Besides, the pharmacist should also ensure that the patient is compliant with the medications. While cardiologists treat the disorder, the role of the pharmacist is critical. Many of these patients are on multiple medications, including antiarrhythmic agents and anticoagulants. In addition, there is some evidence indicating that the use of angiotensin receptor blockers and statins may lower the frequency of atrial fibrillation and increase the probability of successful cardioversion. Thus, the pharmacist has to make sure that the patient's medication doses are therapeutic; there are no drug interactions and that the patient has therapeutic anticoagulation to prevent a stroke (Level V) [12-14].

\section{Outcomes}

Atrial fibrillation prevalence has been on the rise. The risk of stroke is 5-times higher in a patient with known atrial fibrillation compared to the general public. It is estimated that $19.6 \%$ of patients over the age of 65 will have apparent atrial fibrillation by 2030 . The most feared side effect of atrial fibrillation is an acute stroke, which can lead to severe morbidity and mortality. It has been shown that $60 \%$ of strokes secondary to atrial fibrillation can be avoided with the use of anticoagulants. Using the CHADs-2-VASc score to evaluate patients with atrial fibrillation is a helpful guide for the management of these patients with the ultimate goal of preventing stroke. Proper risk factor stratification and medical/surgical therapy can decrease the risk of stroke and heart failure significantly [3]. 


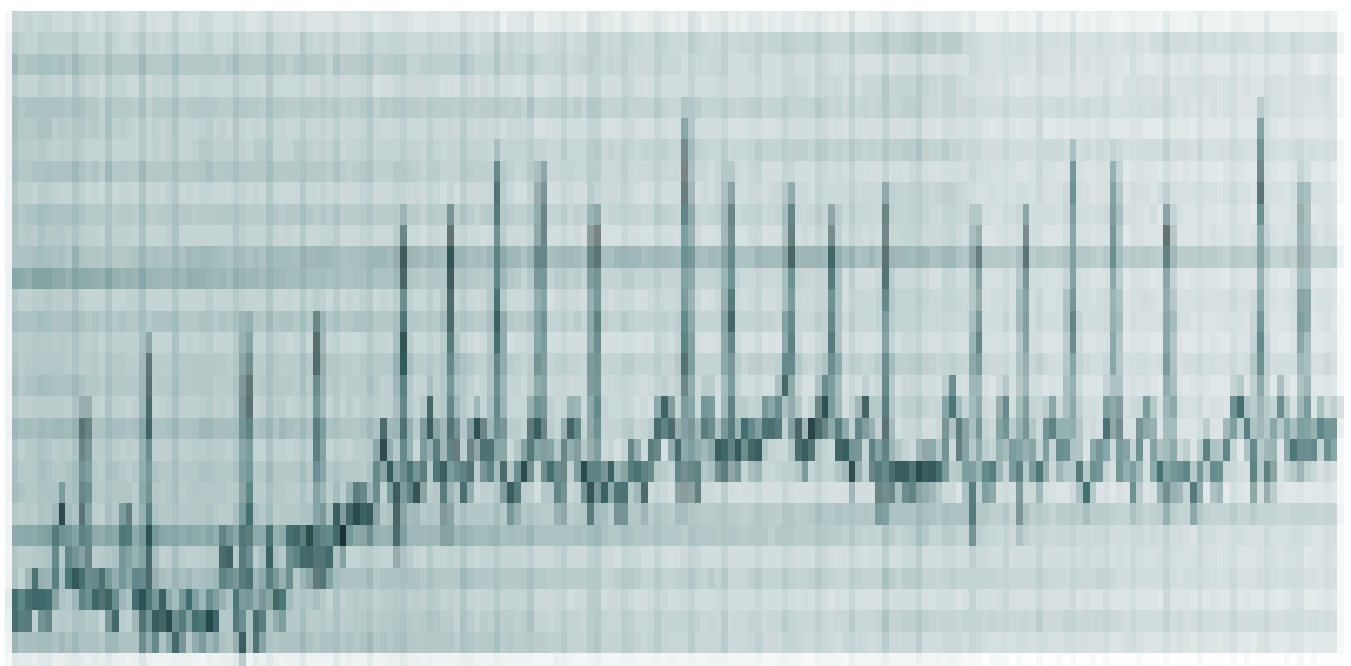

Fig-1: Atrial fibrillation

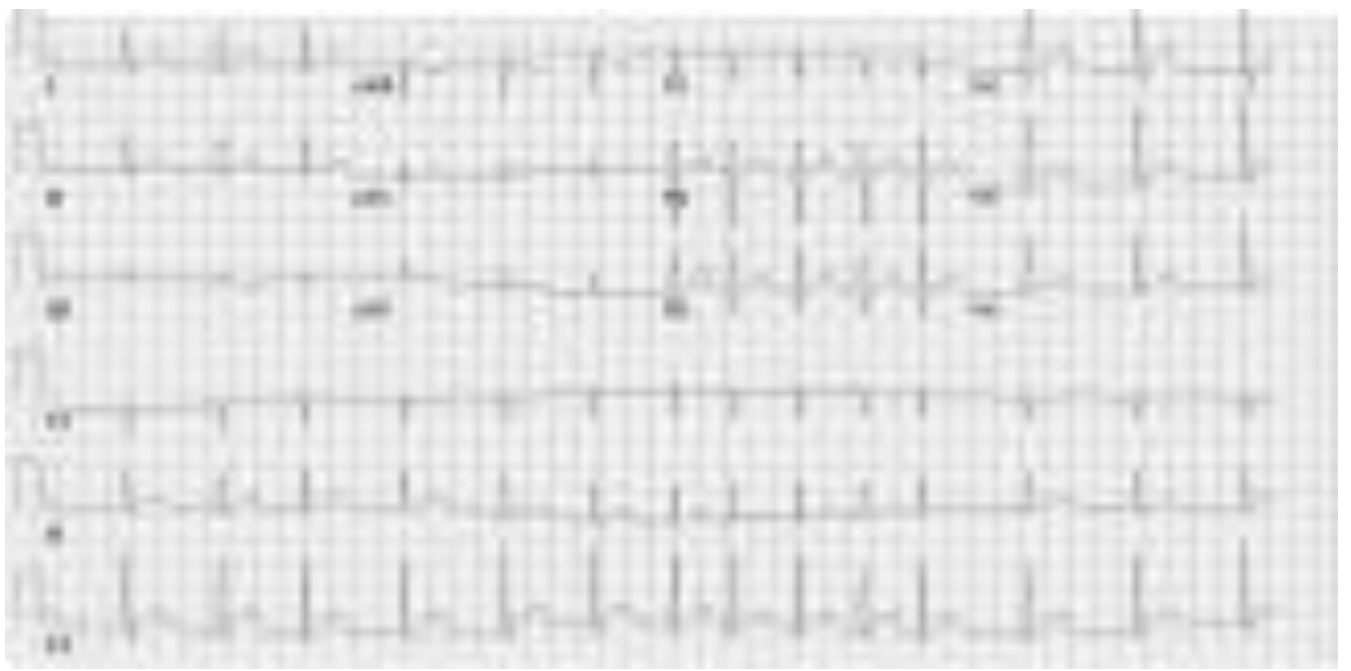

Fig-2: ECG for an atrial fibrillation's case

\section{DISCUSSION}

Atrial fibrillation is the most common sustained tachyarrhythmia in the United States and worldwide. Therapy for atrial fibrillation focuses on 3 goals: minimize thromboembolic stroke risk, control ventricular rate, and control the atrial rhythm in selected patients. Any unstable patient presenting with atrial fibrillation should undergo immediate electrical cardioversion as documented in the Advance Cardiovascular Life Support guidelines. Elective cardioversions are safe and effective with attention to appropriate anticoagulation strategies, including a transesophageal echocardiogram when needed. Treatment strategies involving rate control only and rhythm control are comparable in terms of mortality rates, but many studies support quality-of-life benefits associated with a rhythm-control approach. Thus, rhythm-control is recommended for patients that remain symptomatic despite adequate rate control. Procedurebased treatment for atrial fibrillation, namely catheter ablation, should be considered. Such procedures also include surgical approaches and implantable cardiac devices. Despite increased emphasis on research for AF interventions, a minority of registered AF clinical trials reported the use of PROs as outcome measurements, and very few described collecting validated, AFspecific PROs. With the advent of hybrid procedures for AF ablation, the strengths of surgical and minimally invasive epicardial ablation can be combined with the strengths of catheter based endocardial ablation in a single step procedure to minimize their individual weaknesses. Hybrid (epicardial and endocardial) ablation can be done in a simultaneous or staged manner [16], where simultaneous ablation can limit the potential for unstable iatrogenic flutters which complicate epicardial only ablation. In contrast, staged ablation may allow for maturation of epicardial lesions for evaluation of gaps in ablation lines. Hybrid ablation offer detailed three-dimensional electroanatomical mapping systems and multipolar catheters to ensure adequacy of pulmonary vein isolation, block across lines of ablation as well as targeted ablation for remnant arrhythmias from an incomplete transmural epicardial lesion $[17,18]$. This approach also gives access to areas of the atria not easily accessed epicardially such as the cavotricuspid isthmus, mitral isthmus and interatrial 
septum. Recent studies and systematic literature review [19], conclude that hybrid treatment of lone AF appears to be a safe technique with satisfactory 1-year results and an antiarrhythmic drug-free success rate that is higher than in isolated procedures. These data reflect an under-emphasis of outcome measurements most relevant to patients and among the most important studies in AF. Improving the care of patients with AF will necessitate increased emphasis on these PROs in the pivotal clinical trials of contemporary interventions. Due to the high prevalence of $\mathrm{AF}$, almost every cardiologist and internist have a decent sized patient population with a diagnosis of AF. The initial encounter could occur during an acute and unstable hemodynamic presentation that requires an immediate DCCV then initiation of anticoagulation if not contraindicated. Systematic and detailed evaluation of the patient with stable AF should be implemented including assessment of the risk of thromboembolism, presence of $\mathrm{CHF}$, tachycardia-induced cardiomyopathy, presence of preexcitation, and other comorbidities that will influence the management of AF such as sleep apnea, thyroid disorder, pulmonary disease, obesity, and diabetes mellitus. These AF classifications are not mutually exclusive and it is common for patients with one type of AF to exhibit overlapping features of another type. These classifications are relevant clinically with respect to outcomes and prognosis with rhythm-controlling treatment strategies. Atrial fibrillation is commonly associated with other supraventricular arrhythmias, namely atrial flutter and focal atrial tachycardia.

\section{Conclusion}

In the past few decades, research into $\mathrm{AF}$ has increased tremendously. With it, our understanding of AF pathophysiology and treatment options has improved as well. Over the last 50 years, the FHS and other epidemiological studies have yielded a breadth of data associating various RFs with risk of $\mathrm{AF}$ and providing insight into their mechanistic link to $\mathrm{AF}$ genesis. However, many questions remain. Will genetic studies improve AF risk assessment, identify novel therapeutic targets, and help guide treatment strategies for both primary and secondary prevention of AF. Drugs targeting specific ion channels are approaching the early stages of clinical investigation. There are also new and exciting areas of AF research such as gene therapy, cell therapy and microRNAs and their function on tissue remodelling. With our ageing population and ever increasing prevalence of $\mathrm{AF}$, optimized treatment plans for individuals with AF are essential. However, while advances in technologies have helped elucidated many aspects of AF, many mysteries still remain. With continued research and into $\mathrm{AF}$, we can expect more effective drug therapies and ablation techniques to be developed in the near future. Ongoing and future epidemiological, translational, and clinical studies may provide insight into these unanswered questions and improve clinical outcomes in patients with AF.

\section{REFERENCES}

1. Markides V, Schilling RJ. Atrial fibrillation: classification, pathophysiology, mechanisms and drug treatment. Heart. 2003 Aug; 89(8):939-43.

2. Amin A, Houmsse A, Ishola A, Tyler J, Houmsse M. The current approach of atrial fibrillation management. Avicenna J Med. 2016 Jan-Mar; 6(1):8-16.

3. McManus DD, Rienstra M, Benjamin EJ. An update on the prognosis of patients with atrial fibrillation. Circulation. $2012 \quad$ Sep $\quad 04$ 126(10):e143-6.

4. Mohanty S, Trivedi C, Gianni C, Natale A. Gender specific considerations in atrial fibrillation treatment: a review. Expert Opin Pharmacother. 2018 Mar; 19(4):365-374.

5. Peters SAE, Woodward M. Established and novel risk factors for atrial fibrillation in women compared with men. Heart. 2019 Feb; 105(3):226234.

6. Choi YJ, Choi EK, Han KD, Jung JH, Park J, Lee E, Choe W, Lee SR, Cha MJ, Lim WH, Oh S. Temporal trends of the prevalence and incidence of atrial fibrillation and stroke among Asian patients with hypertrophic cardiomyopathy: A nationwide population-based study. Int J Cardiol. 2018 Dec 15; 273:130-135.

7. Robert R, Porot G, Vernay C, Buffet P, Fichot M, Guenancia C, Pommier T, Mouhat B, Cottin Y, Lorgis L. Incidence, Predictive Factors, and Prognostic Impact of Silent Atrial Fibrillation After Transcatheter Aortic Valve Implantation. Am J Cardiol. 2018 Aug 01; 122(3):446-454.

8. Tarride JE, Quinn FR, Blackhouse G, Sandhu RK, Burke N, Gladstone DJ, Ivers NM, Dolovich L, Thornton A, Nakamya J, Ramasundarahettige C, Frydrych PA, Henein $\mathrm{S}, \mathrm{Ng} \mathrm{K}$, Congdon $\mathrm{V}$, Birtwhistle RV, Ward R, Healey JS. Is Screening for Atrial Fibrillation in Canadian Family Practices Cost-Effective in Patients 65 Years and Older? Can J Cardiol. 2018 Nov; 34(11):15221525 .

9. Karnad A, Pannelay A, Boshnakova A, Lovell AD, Cook RG. Stroke prevention in Europe: how are 11 European countries progressing toward the European Society of Cardiology (ESC) recommendations? Risk Manag Healthc Policy. 2018; 11:117-125.

10. Laäs DJ, Naidoo M. Oral anticoagulants and atrial fibrillation: A South African perspective. S Afr Med J. 2018 Jul 25; 108(8):640-646.

11. Bai CJ, Madan N, Alshahrani S, Aggarwal NT, Volgman AS. Sex Differences in Atrial Fibrillation-Update on Risk Assessment, Treatment, and Long-Term Risk. Curr Treat Options Cardiovasc Med.2018 Aug 27; 20(10):79.

12. Brieger D, Amerena J, Attia JR, Bajorek B, Chan $\mathrm{KH}$, Connell C, Freedman B, Ferguson C, Hall T, 
Haqqani HM, Hendriks J, Hespe CM, Hung J, Kalman JM, Sanders P, Worthington J, Yan T, Zwar NA. National Heart Foundation of Australia and Cardiac Society of Australia and New Zealand: Australian clinical guidelines for the diagnosis and management of atrial fibrillation 2018. Med J Aust. 2018 Oct 15; 209(8):356-362.

13. Pharithi RB, Ranganathan D, O'Brien J, Egom EE, Burke C, Ryan D, McAuliffe C, Vaughan M, Coughlan T, Morrissey E, McHugh J, Moore D, Collins R. Is the prescription right? A review of non-vitamin $\mathrm{K}$ antagonist anticoagulant (NOAC) prescriptions in patients with non-valvular atrial fibrillation. Safe prescribing in atrial fibrillation and evaluation of non-vitamin $\mathrm{K}$ oral anticoagulants in stroke prevention (SAFENOACS) group. Ir J Med Sci. 2019 Feb; 188(1):101-108.

14. Dan GA, Iliodromitis K, Scherr D, Marín F, Lenarczyk R, Estner HL, Kostkiewicz M, Dagres N, Lip GYH. Translating guidelines into practice for the management of atrial fibrillation: results of a European Heart Rhythm Association Survey. Europace. 2018 Aug 01; 20(8):13821387.
15. Wolf PA, Abbott RD, Kannel WB. Atrial fibrillation: A major contributor to stroke in the elderly. The Framingham Study. Arch Intern Med, 1987; 147:1561-1564.

16. La Meir M. New technologies and hybrid surgery for atrial fibrillation. Rambam Maimonides Med J. 2013; 4:e0016.

17. Knecht S, Veenhuyzen G, O'NEILL MD, Wright M, Nault I, Weerasooriya R, Miyazaki S, Sacher F, Hocini M, Jaïs P, Haïssaguerre M. Atrial tachycardias encountered in the context of catheter ablation for atrial fibrillation part II: mapping and ablation. Pacing and clinical electrophysiology. 2009 Apr;32(4):528-38.

18. Kumar N, Bonizzi P, Pison L, Phan K, Lankveld T, Maessen B, La Meir M, Gelsomino S, Maessen J, Crijns H. Impact of hybrid procedure on $\mathrm{P}$ wave duration for atrial fibrillation ablation. Journal of Interventional Cardiac Electrophysiology. 2015 Mar 1;42(2):91-9.

19. Gelsomino S, Van Breugel HN, Pison L, Parise O, Crijns HJ, Wellens F, Maessen JG, La Meir M. Hybrid thoracoscopic and transvenous catheter ablation of atrial fibrillation. European Journal of Cardio-Thoracic Surgery. 2014 Mar 1;45(3):4017. 